\title{
Self-calibration method without joint iteration for distributed small satellite SAR systems
}

\author{
Qing Xu*, Guisheng Liao, Aifei Liu and Juan Zhang
}

\begin{abstract}
The performance of distributed small satellite synthetic aperture radar systems degrades significantly due to the unavoidable array errors, including gain, phase, and position errors, in real operating scenarios. In the conventional method proposed in (IEEE T Aero. Elec. Sys. 42:436-451, 2006), the spectrum components within one Doppler bin are considered as calibration sources. However, it is found in this article that the gain error estimation and the position error estimation in the conventional method can interact with each other. The conventional method may converge to suboptimal solutions in large position errors since it requires the joint iteration between gain-phase error estimation and position error estimation. In addition, it is also found that phase errors can be estimated well regardless of position errors when the zero Doppler bin is chosen. In this article, we propose a method obtained by modifying the conventional one, based on these two observations. In this modified method, gain errors are firstly estimated and compensated, which eliminates the interaction between gain error estimation and position error estimation. Then, by using the zero Doppler bin data, the phase error estimation can be performed well independent of position errors. Finally, position errors are estimated based on the Taylor-series expansion. Meanwhile, the joint iteration between gain-phase error estimation and position error estimation is not required. Therefore, the problem of suboptimal convergence, which occurs in the conventional method, can be avoided with low computational method. The modified method has merits of faster convergence and lower estimation error compared to the conventional one. Theoretical analysis and computer simulation results verified the effectiveness of the modified method.
\end{abstract}

Keywords: Array signal processing, Distributed small satellite synthetic aperture radar (DSS-SAR) systems, Error estimation

\section{Introduction}

With the development of spaceborne synthetic aperture radar (SAR) systems, the functions, such as SAR image, ground moving target indication (GMTI), and SAR interferometry (InSAR), have been well performed [1-4]. In the conventional spaceborne SAR systems, large antennas are required due to the minimum antenna area constraint [5]. However, it leads to the failure for the systems to obtain an image of wide area since the illumination area is inversely related to the aperture size of the antennas [6]. Distributed small satellite synthetic aperture radar (DSSSAR) systems [6-12] were developed to deal with the problem and have received considerable attention in recent years. In DSS-SAR systems, several small satellites

\footnotetext{
* Correspondence: xuqing@mail.xidian.edu.cn

National Laboratory of Radar Signal Processing, Xidian University, Xi'an
} 710071, China

\section{Springer}

(C) 2013 Xu et al.; licensee Springer. This is an Open Access article distributed under the terms of the Creative Commons Attribution License (http://creativecommons.org/licenses/by/2.0), which permits unrestricted use, distribution, and reproduction in any medium, provided the original work is properly cited. move in a special orbital configuration and function as a single "virtual satellite". A small antenna covering wide area is placed on each satellite with the total antenna area constituted by all the small antennas satisfying the minimum antenna area requirement. Due to the flying formation of DSS-SAR systems, along-track baselines and across-track baselines may exist synchronously. And the across-track baseline is needed for terrain height estimation [7] while the along-track baseline is suitable for the function of SAR and GMTI [8]. In [9], the SAR train configuration is analyzed and many scholars focus their attention on SAR imaging or GMTI based on this configuration $[10,11]$. However, for the echo received by each small satellite, range or azimuth (Doppler) ambiguities will occur due to the use of small antennas. In order to image a wide swath unambiguously, the echoes of small antennas should be combined coherently in the DSS-SAR system. 
In $[10,11]$, the low pulse repetition frequency (PRF) is chosen to avoid range ambiguity which results in Doppler ambiguity, and the approaches are also given to suppress Doppler ambiguity. However, their excellent performance is critically dependent on the knowledge of the array manifold (parameterized by many parameters, such as angle of arrivals, and antennas' position information). The array manifold is used in coherent combination of small antennas' echoes to suppress Doppler ambiguity [10]. In practice, there always exist various perturbations in the array manifold which are always called as array errors, such as gain, phase, and position errors. Since the array manifold cannot be exactly obtained because of the existence of array errors, the performance of Doppler ambiguity suppression $[11,12]$ can significantly be degraded. Therefore, it is necessary to estimate and calibrate array errors prior to carry out Doppler ambiguity suppression. In [12], the array error calibration method has also been discussed.In array error estimation of DSS-SAR systems, the existing array calibration methods [13-18] can be applied to the systems only when proper calibration sources are chosen. In [12], spectrum components within one Doppler bin are used as calibration sources with known directions which are called "virtual calibration sources." Since the number of spectrum components is more than one due to Doppler ambiguity, it is possible to apply array calibration methods with more than one calibration source [15-18] to DSS-SAR systems. In [12], a two-step iterative auto-calibration method is presented to estimate gain-phase and position errors. In the first step, assuming that position errors are known, gain-phase errors are estimated by using the method in [16]. In the second step, based on the gain-phase error estimated in the former step, position errors can be obtained by the least squared method [17]. These two steps should be iterated alternatively to obtain final solutions. For convenience, the array error estimation method in [12] is named as the conventional method (the comparison in this article is limited in terms of array error estimation method).

In the conventional method, two kinds of errors, the gain-phase error and the position error, are respectively estimated under the assumption that other kinds of errors are known. The inherent relationship among gain, phase, and position errors is not considered and analyzed in the conventional method. In this article, by studying of the conventional method, the following two aspects are observed. First, gain error estimation and position error estimation can affect each other, which will influence the convergence rate. And the conventional method may even suffer from suboptimal convergence in large position errors. Second, if spectrum components within the zero Doppler bin are used as calibration sources to estimate the errors, phase error estimation can be performed independent of position errors. Based on the above two aspects, a modified array error estimation method is proposed in this article. First, in order to eliminate the interaction between the gain and position error estimations, gain errors are first estimated and compensated. Then, phase errors are estimated by using the spectrum components within the zero Doppler bin as calibration sources. Finally, position errors are estimated based on Taylor-series expansion. However, since Taylor-series expansion causes approximation errors, position error estimation should be iterated in order to obtain higher estimation accuracy. In comparison with the conventional method, the modified method can avoid the iteration between gain-phase error estimation and position error estimation, which guarantees that it can converge to optimal solutions with lower computational load and fast convergence speed. Simulation results verify that the modified method performs better than the conventional one.

The remainder of the article is arranged as follows. In Section 2, the modified method for DSS-SAR systems is described in detail. The performance of the modified method is verified by using Monte Carlo simulations in Section 3. The article ends with some conclusions given in Section 4.

\section{Modified method for DSS-SAR systems}

\subsection{Signal model}

In this article, the signal model of DSS-SAR systems will be introduced briefly as follows [12]. The SAR train configuration in which all the satellites are arranged along the $X$-axis as an array is given in Figure 1 . The $(X, Y, Z)$ direction is referred to as (along-track, cross-track, and radial). The array operates in the side-looking strip mode and all satellites have an identical along-track velocity, denoted by $v_{s} . \theta, \varphi$, and $\phi$ represent azimuth angle, incidence angle, and cone angle, respectively. Azimuth angle $\theta\left(\tau, f_{d}\right)$ can be expressed as $\theta\left(\tau, f_{d}\right)=\theta_{o}+\Delta \theta\left(\tau, f_{d}\right)$, where

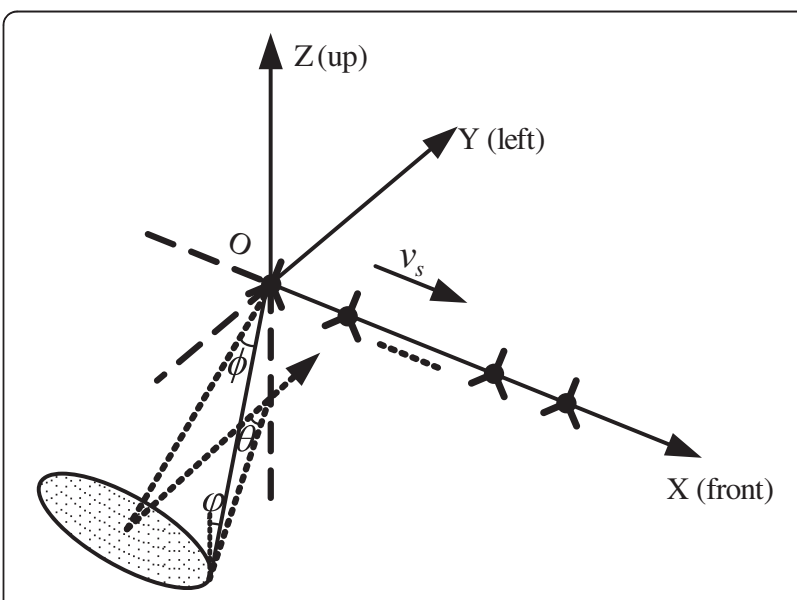

Figure 1 Configuration of the DSS-SAR array. 
$\theta_{o}$ and $\Delta \theta\left(\tau, f_{d}\right)$ are the azimuth angle and the offset from the beam center, respectively. $\tau$ denotes the fast time corresponding to the range bin and $f_{d}$ denotes the Doppler frequency which corresponds to the slow time.

True coordinates, measured coordinates, and corresponding position errors of the $m$ th satellite are denoted by $\left(x_{m}, y_{m}\right.$, $\left.z_{m}\right),\left(x_{m o}, y_{m o}, z_{m o}\right)$, and $\left(\Delta x_{m}, \Delta y_{m}, \Delta z_{m}\right)$, respectively. The relationship among them is

$$
\left(x_{m}, y_{m}, z_{m}\right)=\left(x_{m o}, y_{m o}, z_{m o}\right)+\left(\Delta x_{m}, \Delta y_{m}, \Delta z_{m}\right),
$$

where $y_{m o}=0, z_{m o}=0$. The first satellite is taken as reference, i.e., $\left(\Delta x_{1}, \Delta y_{1}, \Delta z_{1}\right)=(0,0,0)$ is imposed. Then the clutter echo in the range-Doppler domain received by the $m$ th satellite after demodulation in the strip mode and broadside geometry can be written as [12]

$$
S_{m}\left(\tau, f_{d}\right)=g_{m} \mathrm{e}^{j \xi_{m}} \sum_{i=-I}^{I} \mathrm{e}^{j \frac{4 \pi}{\lambda} d_{m}\left(\tau, f_{d}+i f_{r}\right)} H_{m}\left(\tau, f_{d}+i f_{r}\right)+n_{m}\left(\tau, f_{d}\right),
$$

where $g_{m}$ and $\xi_{1}$ are the gain and phase error of the $m$ th array element relative to the first array element (as such, $g_{1}=1$ and $\left.\xi_{1}=0\right)$. $\lambda$ denotes the wavelength of the carrier. $n_{m}\left(\tau, f_{d}\right)$ is the additive white Gaussian noise. $2 I+1$ is the number of spectrum components within one rangeDoppler bin. The spectrum components have different azimuth angles $\theta\left(\tau, f_{d}+i f_{r}\right)$ and the same incidence angles $\varphi(\tau)$ which can be expressed as follows:

$$
f_{d}+i f_{r}=\frac{2 v_{s}}{\lambda} \sin \theta\left(\tau, f_{d}+i f_{r}\right) \sin \varphi(\tau), \quad i=-l, \ldots, l .
$$

In the following, for simplicity, $(\cdot)^{i}$ is used to denote the variable associated with $\left(f_{d}+i f_{r}\right)$, such as $\theta^{i}$ denotes $\theta\left(\tau, f_{d}+i f_{r}\right) . d_{m}^{i}=x_{m} \sin \theta^{i} \sin \varphi(\tau)+y_{m} \cos \theta^{i} \sin \varphi(\tau)+z_{m}$ $\cos \phi(\tau)$. In the side-looking strip mode, $\theta_{o}=0$ and then $\theta^{i}=\Delta \theta^{i}$. Moreover, $\Delta \theta^{i}$ is very small $\left(\left|\Delta \theta^{i}\right| \leq 0.43^{\circ}\right.$ for a small antenna with the azimuth length of $2 \mathrm{~m}$ at $X$-band) in practice. Thus, $\cos \theta^{i}=\cos \Delta \theta^{i} \approx 1$ can be obtained with small angle approximation. Since $y_{m o}=z_{m o}=0$,

$$
\begin{aligned}
d_{m}^{i}= & \left(x_{m o}+\Delta x_{m}\right) \sin \theta^{i} \sin \varphi(\tau)+\left(y_{m o}+\Delta y_{m}\right) \cos \theta^{i} \sin \varphi(\tau) \\
& +\left(z_{m o}+\Delta z_{m}\right) \cos \varphi(\tau) \approx\left(x_{m o}+\Delta x_{m}\right) \sin \theta^{i} \sin \varphi(\tau) \\
& +\Delta y_{m} \sin \varphi(\tau)+\Delta z_{m} \cos \varphi(\tau)
\end{aligned}
$$

$H_{m}\left(\tau, f_{d}\right)$ is the complex envelope of the clutter echo in the range-Doppler domain,

$$
H_{m}\left(\tau, f_{d}\right)=\iint \sigma(x, y) h\left(\tau-\frac{2 r_{m}\left(x, y, z, f_{d}\right)}{c}\right) G\left(f_{d}\right) e^{-j \Psi^{\prime}\left(x, y, z f_{d}\right)} d x d y
$$

where $\sigma(x, y)$ is the complex surface scattering coefficient. $\Psi^{\prime}\left(x, y, z, f_{d}\right)=\frac{4 \pi}{\lambda} \sqrt{y^{2}+z^{2}}-\frac{\pi \lambda \sqrt{y^{2}+z^{2}}}{2 v_{s} 2}+2 \pi f_{d} \frac{x}{v_{s}} \cdot h$ $(\tau)$ is a linear frequency modulated signal. $G\left(f_{d}\right)$ denotes the Fourier transform of the function which represents the antenna pattern and other time-variant characters (identical to all receiving antennas). $r_{m}\left(x, y, z, f_{d}\right)$ is the slant range from the $m$ th satellite to the ground cell,

$$
\begin{aligned}
r_{m}\left(x, y, z, f_{d}\right) & =\sqrt{y^{2}+z^{2}}+\frac{\lambda^{2} \sqrt{y^{2}+z^{2}} f_{d}^{2}}{8 v_{s}^{2}} \\
& -\Delta y_{m} \cos \theta\left(\tau, f_{d}\right) \sin \varphi(\tau) \\
& -\Delta z_{m} \cos \varphi(\tau) .
\end{aligned}
$$

Since $\Delta y_{m} \cos \theta\left(\tau, f_{d}\right) \sin \varphi(\tau)$ and $\Delta z_{m} \cos \varphi(\tau)$ can be neglected in $r_{m}\left(x, y, z, f_{d}\right)$, it can be obtained that $r_{1}(x, y$, $\left.z, f_{d}\right) \approx \cdots \approx r_{M}\left(x, y, z, f_{d}\right) \triangleq r\left(x, y, z, f_{d}\right)$. So, as $H_{1}\left(\tau, f_{d}\right) \approx$ $\cdots \approx H_{M}\left(\tau, f_{d}\right) \triangleq H\left(\tau, f_{d}\right)$.

Based on the previous analysis, (2) can be rewritten as

$$
S_{m}\left(\tau, f_{d}\right)=g_{m} \mathrm{e}^{j \xi_{m}^{\prime}} \sum_{i=-I}^{I} e^{j \frac{4 \pi}{\lambda} d^{\prime} m^{i}} H^{i}+n_{m}\left(\tau, f_{d}\right)
$$

where

$$
\begin{aligned}
\xi_{m}^{\prime} & =\xi_{m}+\frac{4 \pi}{\lambda}\left(\Delta y_{m} \sin \phi(\tau)+\Delta z_{m} \cos \varphi(\tau)\right), \\
d_{m}^{\prime}{ }^{i} & =\left(x_{m o}+\Delta x_{m}\right) \sin \theta^{i} \sin \varphi(\tau)=\frac{\lambda}{2 v_{s}}\left(f_{d}+i f_{r}\right)\left(x_{m o}+\Delta x_{m}\right) .
\end{aligned}
$$

It is found that the contributions of position errors to array outputs can be separated into two parts. One part associated with cross-track and radial position errors is fixed for all clutter echoes and can be regarded as array phase errors. The other part related to along-track position errors changes with spectrum components. For simplicity, the total phase error $\xi_{m}^{\prime}$ is called the phase error and the along-track position error is named as the position error in the following. The unknown errors considered here are the position error $\Delta x_{m}(m=2, \ldots, M)$ and the gain-phase error $g_{m} e^{j \xi^{\prime}}{ }_{m} \quad(m=2, \ldots, M)$. Using vector notation, Equation (7) can be rewritten as follows

$$
S=\Gamma A H+n
$$

where $\mathbf{S}=\left[S_{1}, \ldots, S_{M}\right]^{T}, \quad \boldsymbol{\Gamma}=\operatorname{diag}\left\{1, \ldots, g_{M} e^{j \xi_{M}^{\prime}}\right\}, \mathbf{A}=$ $\left[\mathbf{a}^{-I}, \ldots, \mathbf{a}^{I}\right], \mathbf{a}^{i}=\left[1, \ldots, e^{j \frac{4 \pi}{\lambda} d^{i i}}\right]^{T}, \mathbf{H}=\left[H^{-I}, \ldots, H^{I}\right]^{T},(\bullet)^{T}$ denotes the transpose operation.

The covariance matrix of $\mathbf{S}$ is denoted as $\mathbf{R}_{\mathbf{S S}}$. Denote eigenvalues and corresponding eigenvectors of the covariance matrix $\mathbf{R}_{\mathbf{S S}}$ with $\chi_{m}$ (listed in descending order) and $\mathbf{u}_{m}(m=1, \ldots, M)$. Each column of $\boldsymbol{\Gamma} \mathbf{A}$ is orthogonal to the matrix $\mathbf{U}=\left[\mathbf{u}_{2 I+2}, \ldots, \mathbf{u}_{M}\right]$. And the orthogonality can be used to estimate the gain-phase and position errors. 


\subsection{The conventional method}

According to [12], the cost function to estimate gainphase errors is

$$
J_{p}=\sum_{i=-I}^{I}\left\|\mathbf{U}^{H} \boldsymbol{\Gamma} \mathbf{a}^{i}\right\|^{2}
$$

where $(\cdot)^{H}$ represents the conjugate transpose operation. The cost function is given based on the MUSIC approach. If the true $\Gamma$ and $\mathbf{a}^{i}$ are obtained, $J_{p}$ should be zero. Minimizing $J_{p}$, the estimation of $\Gamma$ and $\mathbf{a}^{i}$ can be achieved. Thus, $\Delta(\Delta=\operatorname{diag}\{\boldsymbol{\Gamma}\})$, gain-phase errors, can be obtained by

$$
\lambda=\mathbf{Q}^{-1} \mathbf{w} / \mathbf{w}^{T} \mathbf{Q}^{-1} \mathbf{w}
$$

where

$$
\begin{aligned}
& \mathbf{w}=[1,0, \ldots, 0]^{T}, \\
& \mathbf{Q}=\sum_{i=-I}^{I}\left(\mathbf{D}^{i}\right)^{H} \mathbf{U} \mathbf{U}^{H} \mathbf{D}^{i}, \\
& \mathbf{D}^{i}=\operatorname{diag}\left\{\mathbf{a}^{i}\right\} .
\end{aligned}
$$

To estimate position errors, $e^{j \frac{4 \pi}{\lambda} d^{\prime i}} \quad(m=1, \ldots, M)$ is expanded using Taylor-series based on (3):

$$
\begin{aligned}
e^{j \frac{4 \pi}{\lambda} d_{m}^{\prime i}} & \approx e^{j \frac{4 \pi}{\lambda} x_{m o} \sin \Delta \theta^{i} \sin \varphi(\tau)}\left(1+j \frac{4 \pi}{\lambda} \Delta x_{m} \sin \Delta \theta^{i} \sin \varphi\right) \\
& \approx e^{j \frac{2 \pi}{v_{s}}\left(f_{d}+i f_{r}\right) x_{m o}}\left(1+j \frac{2 \pi}{v_{s}}\left(f_{d}+i f_{r}\right) \Delta x_{m}\right)
\end{aligned}
$$

Then, based on (3) and (14), $\mathbf{a}^{i}$ can be rewritten as

$$
\mathbf{a}^{i}=\mathbf{a}_{o}^{i}+\mathbf{a}_{\Delta}^{i} \Delta \mathbf{X}
$$

where

$$
\begin{aligned}
& a_{o}^{i}=\left[1, e^{j \frac{2 \pi}{v_{s}}\left(f_{d}+i f_{r}\right) x_{2 o}}, \ldots, e^{j \frac{2 \pi}{v_{s}}\left(f_{d}+i f_{r}\right) x_{M o}}\right]^{T} \\
& \mathbf{a}_{\Delta}^{i}=\operatorname{diag} \\
& \quad\left\{0, j \frac{2 \pi}{v_{s}}\left(f_{d}+i f_{r}\right) e^{j \frac{2 \pi}{v_{s}}\left(f_{d}+i f_{r}\right) x_{2 o}}, \ldots, j \frac{2 \pi}{v_{s}}\left(f_{d}+i f_{r}\right) e^{j \frac{2 \pi}{v_{s}}\left(f_{d}+i f_{r}\right) x_{M o}}\right\}
\end{aligned}
$$

$$
\Delta \mathbf{X}=\left[1, \Delta x_{2}, \ldots, \Delta x_{M}\right]^{T}
$$

Therefore, the cost function (9) can be modified as

$$
J_{c}=\|\mathbf{E}-\mathbf{F} \Delta \mathbf{X}\|^{2}
$$

where $\quad \mathbf{E}=\left[\mathbf{e}_{-I}^{T} \ldots \mathbf{e}_{I}^{T}\right]^{T}, \quad \mathbf{F}=\left[\mathbf{f}_{-I}^{T} \ldots \mathbf{f}_{I}^{T}\right]^{T}, \quad \mathbf{e}_{i}=\mathbf{U}^{H} \boldsymbol{\Gamma} \mathbf{a}_{o}^{i}, \quad \mathbf{f}_{i}=-$ $\mathbf{U}^{H} \mathbf{\Gamma} \mathbf{a}_{\Delta}^{i}$. The position error $\Delta \mathbf{X}$ is the least squared solution of (19)

$$
\Delta \mathbf{X}=\operatorname{real}\left\{\left(\mathbf{F F}^{H}\right)^{-1} \mathbf{F}^{H} \mathbf{E}\right\}
$$

where real $(\bullet)$ takes the real part of the matrix. The phase error introduced by Taylor-series expansion above is within $4^{\circ}$ at $X$-band under the assumption that $\Delta x_{m} \leq$ $20 \mathrm{~cm}$. And higher accuracy can be achieved after several iterations. However, from computer simulations, it is found that if $\Delta x_{m}>30 \mathrm{~cm}$, the estimate difference of position errors will be larger than $0.008 \mathrm{~m}$. The conventional method can be summarized as joint iteration between the following two steps.

- Step 1 Choose some Doppler bin to obtain gainphase errors based on (10).

- Step 2 Estimate position errors based on (20) and compensate $x_{m o}$ by $\Delta x_{m}$.

\subsection{Formulation of the modified method}

Lets consider the gain error estimation of the conventional method.

Based on (12) and (13), we obtain

$$
\mathbf{Q}=\sum_{i=-I}^{I}\left(\mathbf{C}^{i}\right)^{H} \odot\left(\mathbf{U} \mathbf{U}^{H}\right) \odot \mathbf{C}^{i}=\left\{\sum_{i=-I}^{I}\left(\mathbf{C}^{i}\right)^{H} \odot \mathbf{C}^{i}\right\} \odot\left(\mathbf{U} \mathbf{U}^{H}\right)
$$

where $\mathbf{C}^{i}=\left[\begin{array}{ccc}\mathbf{D}_{11}^{i} & \cdots & \mathbf{D}_{M M}^{i} \\ \vdots & \ddots & \vdots \\ \mathbf{D}_{11}^{i} & \cdots & \mathbf{D}_{M M}^{i}\end{array}\right], \odot$ denotes dot product (i.e., element-wise multiplication). With the definition of $\mathbf{Z}^{i}=\left(\mathbf{C}^{i}\right)^{H} \odot \mathbf{C}^{i}$ and $\mathbf{Z}=\sum_{i=-I}^{I} \mathbf{Z}^{i}=\sum_{i=-I}^{I}\left(\mathbf{C}^{i}\right)^{H} \odot \mathbf{C}^{i}$

can be rewritten as

$$
\mathbf{Q}=\mathbf{Z} \odot\left(\mathbf{U} \mathbf{U}^{H}\right)
$$

The element in the $k$ th row and the $l$ th column of the matrix $\mathbf{Z}^{i}$ and $\mathbf{Z}$ can be obtained, respectively, as

$$
\mathbf{Z}_{k l}^{i}=\left(\mathbf{D}_{k k}^{i}\right)^{*} \odot \mathbf{D}_{l l}^{i}=e^{j \frac{4 \pi}{v_{s}}\left(f_{d}+i f_{r}\right)\left(x_{l o}+\Delta x_{l}-x_{k o}-\Delta x_{k}\right)} .
$$




$$
\begin{aligned}
\mathbf{Z}_{k l} & =\sum_{i=-I}^{I} \mathbf{Z}_{k l}^{i}=e^{j \frac{4 \pi}{v_{s}} f_{d}\left(x_{l o}+\Delta x_{l}-x_{k o}-\Delta x_{k}\right)} \\
& \sum_{i=-I}^{I} e^{j \frac{4 \pi}{v_{s}} i f_{r}\left(\left(x_{l o}-x_{k o}\right)+\left(\Delta x_{l}-\Delta x_{k}\right)\right)} \\
& =e^{j \frac{4 \pi}{v_{s}} f_{d}\left(x_{l o}+\Delta x_{l}-x_{k o}-\Delta x_{k}\right)} \\
& \left(1+\sum_{i=1}^{I} \cos \left(\frac{4 \pi}{v_{s}} i f_{r}\left(\left(x_{l o}-x_{k o}\right)+\left(\Delta x_{l}-\Delta x_{k}\right)\right)\right)\right)
\end{aligned}
$$

Based on (24), $\mathbf{Z}$ is a function of the position error $\Delta \mathbf{X}$

$$
\mathbf{Z}=f(\Delta \mathbf{X})
$$

And $\mathbf{Q}$ is also parameterized by the position error $\Delta \mathbf{X}$

$$
\mathbf{Q}=f(\Delta \mathbf{X})
$$

In [12], Equation (10) is used to estimate gain errors, which means

$$
\mathbf{g}=\operatorname{abs}\left(\mathbf{Q}^{-1} \mathbf{w} /_{\mathbf{w}^{T}} \mathbf{Q}^{-1} \mathbf{w}\right)
$$

where $\mathbf{g}=\left[1, g_{2}, \ldots, g_{M}\right]^{T}$, abs $(\bullet)$ denotes the complex modulus of the elements of a matrix. The gain error estimate, $\hat{\mathbf{g}}$, will be inaccurate because of the existence of the position error $\Delta \mathbf{X}$ (derived by $\mathbf{Q}$ ). Moreover, the estimation differences of gain errors will be larger with the increase of position errors.

However, the estimate differences of gain errors can also influence the estimation accuracy of position errors during the second step in the conventional method. It is analyzed as follows. $\sim^{i}$ can be rewritten as

$$
\sim^{i}=\boldsymbol{\rho} e^{j \boldsymbol{\eta}}
$$

where $\boldsymbol{\rho}=\left[\rho_{1}, \ldots, \rho_{M}\right]^{T}$ and $\boldsymbol{\eta}=\left[\eta_{1}, \ldots, \eta_{M}\right]^{T}$ denote the amplitude and phase of $\sim \mathbf{a}^{i}$, respectively.

Since (15) is obtained through the Taylor-series expansion, $\rho_{m}(m=2, \ldots, M)$ approaches one but not exactly equals one. Considering the $i$ th "virtual calibration sources," the following formula can be obtained

$$
J_{c}^{i}=\left\|\mathbf{U}^{H} \boldsymbol{\Gamma} \mathbf{a}^{i}\right\|^{2} \approx\left\|\mathbf{U}^{H} \boldsymbol{\Gamma}^{\sim} \mathbf{a}^{i}\right\|^{2}
$$

Based on the solution obtained by (10), the element in the $k$ th row and the $k$ th column of $\Gamma^{\sim} \mathbf{a}^{i}$ (which is a diagonal matrix) can be expressed as

$$
\begin{aligned}
\left(\boldsymbol{\Gamma}^{\sim} \mathbf{a}^{i}\right)_{k k} & =g_{k} e^{j \xi^{\prime}} \rho_{k} e^{j \eta_{k}} \\
& =\left(\hat{g_{k}}+\Delta g_{k}\right) e^{j\left(\hat{\xi^{\prime}}+\Delta \xi_{k}^{\prime}\right)} \rho_{k} e^{j \eta_{k}} \\
& =\hat{g_{k}} e^{j \xi_{k}^{\prime}} \rho_{k} \hat{g_{k}}+\Delta g_{k} \hat{g_{k}} e^{j\left(\eta_{k}+\Delta \xi^{\prime}\right)} \\
& =\hat{g_{k}} e^{j \xi^{\prime}} \hat{\rho}_{k} e^{j \hat{\eta}_{k}}
\end{aligned}
$$

where $\hat{g_{k}}$ and $\Delta g_{k}$ denote estimated values and differences of gain errors, $\hat{\xi}_{k}^{\prime}$ and $\Delta \xi_{k}^{\prime}$ represent estimated values and differences of phase errors, respectively. Then $\widehat{\rho_{k}}=\rho_{k}\left(\widehat{g_{k}}+\Delta g_{k}\right) / \widehat{g_{k}}$ and $\hat{\eta}_{k}=\eta_{k}+\Delta \xi_{k}^{\prime}$ instead of $\rho_{k}$ and $\eta_{k}$ will be obtained if (29) is used to estimate position errors. Thus, $\Delta g_{k}$ will affect the position error estimation accuracy. Considering the fact that position errors have an effect on the gain error estimation during the first step while gain errors affect position error estimation during the second step, estimating differences of gain and position errors using (27) and (20) iteratively in the conventional method could converge to suboptimal solutions in large position errors.

So, gain errors should be estimated first and then compensated. Here, the method in [18] is used to estimate gain errors

$$
\hat{g_{m}}=\frac{1}{P} \sum_{f_{d}} \sqrt{\frac{\left(\mathbf{R}_{\mathbf{S S}}\left(f_{d}\right)\right)_{m m}-\sigma_{n}^{2}}{\left(\mathbf{R}_{\mathbf{S S}}\left(f_{d}\right)\right)_{11}-\sigma_{n}^{2}}} \quad(m=1, \ldots, M),
$$

where $P$ is the number of Doppler bins, $\sigma_{n}^{2}$ can be obtained by averaging $\chi_{2 I+2}$ to $\chi_{M}$.

After estimating gain errors, phase and position error estimations will be considered in the following. The conventional method requires joint iteration between phase and position error estimations. Through the following study, the joint iteration is avoided in the modified method.

In the certain Doppler bin $f_{d}=0$, there exist $2 I+1$ spectrum components which are $i f_{n} i=-I, \ldots, I$, due to the existence of Doppler ambiguity. Based on (13), we obtain

$$
\mathbf{D}^{-i}=\left(\mathbf{D}^{i}\right)^{*}
$$

Hence, $\mathbf{C}^{-i}=\left(\mathbf{C}^{i}\right)^{*}$ is obtained and so as the following equation

$$
\begin{aligned}
\mathbf{Z}^{-i} & =\left(\left(\mathbf{C}^{-i}\right)^{H} \odot \mathbf{C}^{-i}\right)=\left(\left(\left(\mathbf{C}^{i}\right)^{*}\right)^{H} \odot\left(\mathbf{C}^{i}\right)^{*}\right) \\
& =\left(\left(\mathbf{C}^{i}\right)^{H} \odot \mathbf{C}^{i}\right)^{*}=\left(\mathbf{Z}^{i}\right)^{*} .
\end{aligned}
$$


Thus, $\mathbf{Z}$ can be obtained as

$$
\mathbf{Z}=\sum_{i=1}^{i} 2 \operatorname{real}\left(\mathbf{Z}^{i}\right)+\operatorname{real}\left(\mathbf{Z}^{0}\right)=\sum_{i=-I}^{i} \operatorname{real}\left(\mathbf{Z}^{i}\right) .
$$

Based on (21) and (34), the phase of $\mathbf{Q}$ behaves independently of position errors. Hence, using the data of the zero Doppler bin, phase errors can directly be obtained without the influence of existing position errors by the following estimator

$$
\xi^{\prime}=\operatorname{angle}\left(\mathbf{Q}^{-1} \mathbf{w} /_{\mathbf{w}^{T}} \mathbf{Q}^{-1} \mathbf{w}\right)
$$

where $\hat{\xi}^{\prime}=\left[\xi_{1}^{\prime}, \xi_{2}^{\prime}, \ldots, \xi_{M}^{\prime}\right]^{T}$, angle(.) returns the phase angles of a matrix. This result is consistent with the phenomenon mentioned in [12] that when $f_{d}=0$ is chosen, the estimation accuracy of phase errors can be satisfactory with position errors assumed to be zero. Since $\Delta \xi_{k}^{\prime}$ is induced without the effect of position errors, we do not discuss the influence of $\Delta \xi_{k}^{\prime}$ on the estimation of the position error, $\Delta \mathbf{X}$, described by (30).

Based on the analysis above, the modified method can be summarized as follows.

- Step 1 Estimate gain errors based on (31) and compensate the received data using estimated gain errors.

- Step 2 Choose the zero Doppler bin to obtain phase error estimate $\hat{\xi}^{\prime}$ based on (35).

- Step 3 Use $\hat{\xi}^{\prime}$ to reconstruct $\sim=\operatorname{diag}\left\{e^{j \widehat{\xi}^{\prime}}\right\}$ and estimate position errors based on (20).

Through the analysis above, phase error estimation method can perform robustly with the existence of position errors. Since the Taylor-series expansion is used in estimating position errors, Step 3 should be applied repeatedly in order to obtain higher estimation accuracy with $x_{m o}$ compensated by $\Delta x_{m}$ (which is estimated in the former iteration) in each iteration.

In the modified method, gain errors are compensated first, hence the relationship between gain and position error estimations is eliminated, which guarantees the estimation accuracy.

The change on the iterative fashion makes it possible for the modified method to work with less computational load. Let $L$ denote the iteration number for the conventional method, the computational load is $3 L M^{3}$ which mainly arises from the matrix eigendecomposition (equals $M^{3}$ ) and the matrix inversion (equals $M^{3}$ ). However, the computational load of the modified method is $2 M^{3}+L^{\prime} M^{3}$ in which $L^{\prime}$ denotes the iteration number of Step 3. From the computer simulations, we find that the estimates of the gain, phase, and position errors through the conventional method do not converge to the final solutions even when $L$ equals to be 10 and the zero Doppler bin is chosen. And for the modified method, $L^{\prime}$ should be more than two to satisfy the estimation accuracy. For example, based on the assumptions that $L=10$ and $L^{\prime}=3$, the computational load of the modified algorithm is $5 \mathrm{M}^{3}$, which is lower than that of the conventional method $\left(30 M^{3}\right)$.

\section{Simulation experiment}

To verify the effectiveness of the modified method, computer simulations are given in this section. Experimental results are obtained based on 200 trials. The simulated DSS-SAR system in the side-looking mode is composed by seven satellites and all the satellites distribute uniformly with the antenna spacing $l=v_{s} T_{r} / M\left(T_{r}\right.$ is the pulse repetition period) in the along-track direction $[9,12]$. The other parameters are listed in Table 1 . The gain, phase, and position errors for each of the $M$ satellites, i.e., $g_{m}, \xi_{m}^{\prime}$, and $\Delta x_{m}$, are defined as uniform random variables in $[1-\alpha, 1+\alpha],[-\pi, \pi]$, and $[-\Delta \mathrm{p}$, $\Delta \mathrm{p}]$, respectively. They are generated and maintained invariant in each trial. The average root mean squared error (ARMSE) of gain, phase, and position errors are defined as $\frac{1}{M-1} \sum_{m=2}^{M} \operatorname{RMSE}\left\{g_{m}\right\}, \frac{1}{M-1} \sum_{m=2}^{M} \operatorname{RMSE}\left\{\xi_{m}^{\prime}\right\}$, $\frac{1}{M-1} \sum_{m=2}^{M} \operatorname{RMSE}\left\{\Delta x_{m}\right\}$, respectively. $L$ and $L^{\prime}$ are set to be 10 and 3 , respectively. The Doppler frequency $f_{d}$ is fixed to be zero.

\subsection{Effect of SNR}

Suppose $\Delta p=0.25 l$ and $\alpha=0.2$. The ARMSE curves of gain, phase, and position error estimates versus signal-to -noise ratio (SNR) are shown in Figure $2 \mathrm{a}-\mathrm{c}$, respectively. From Figure 2, it is observed that both the modified method and the conventional method perform better as the SNR increases. From Figure 2a,c, it can be seen that gain and position error estimation performances of the conventional method are worse than those of the modified method. It is because gain

\section{Table 1 Simulation parameters of DSS-SAR systems}

\begin{tabular}{ll}
\hline Orbit altitude & $750 \mathrm{~km}$ \\
Incidence angle & $45^{\circ}$ \\
Band & $X$-band \\
Antenna size & $2 \mathrm{~m} \times 1 \mathrm{~m}$ \\
Bandwidth & $100 \mathrm{MHz}$ \\
PRF & $1496 \mathrm{~Hz}$ \\
Velocity & $7481.5 \mathrm{~m} / \mathrm{s}$ \\
\hline
\end{tabular}




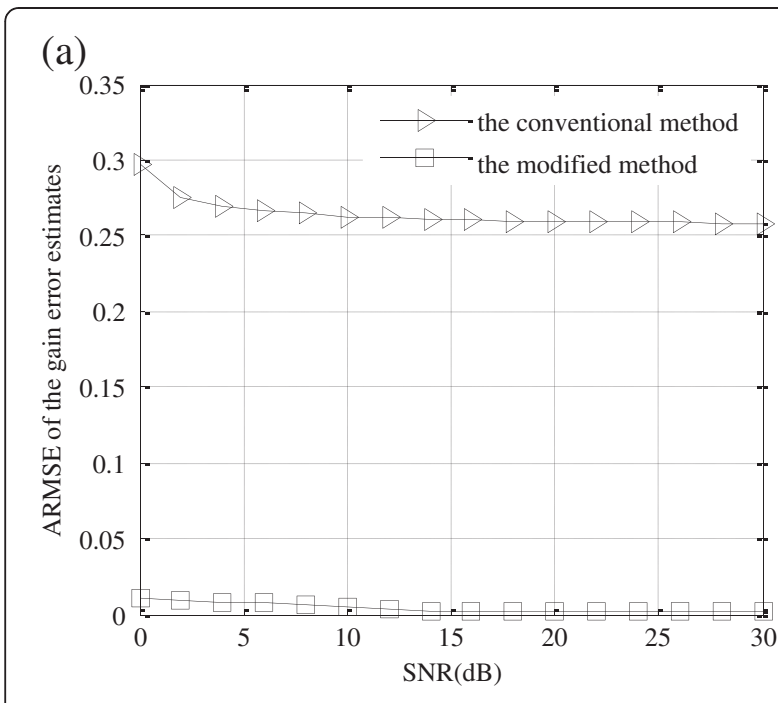

(b)

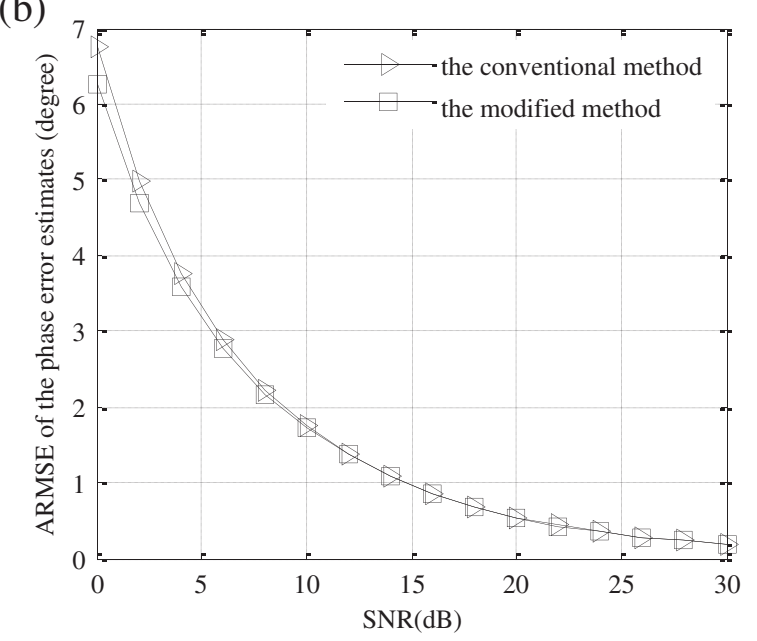

(c)

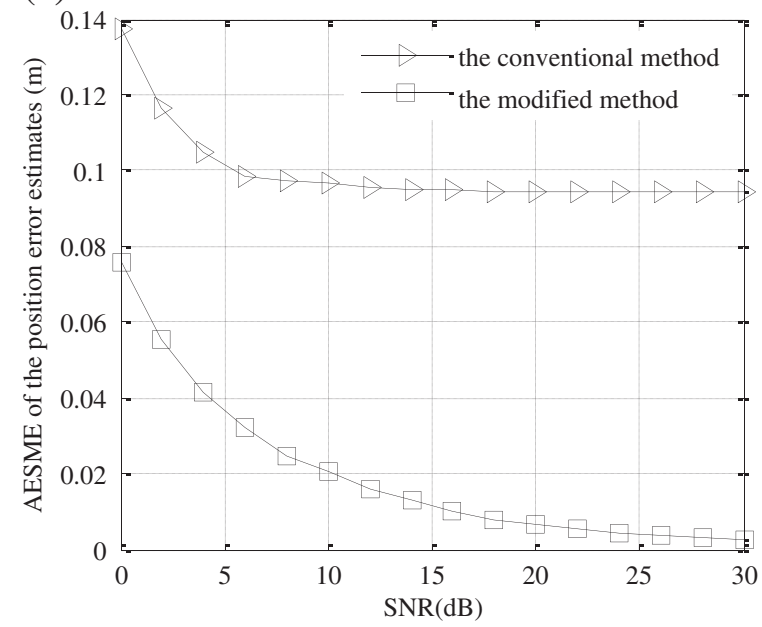

Figure 2 ARMSE of gain, phase and position error estimates versus SNR. (a) ARMSE of gain error estimates versus SNR; (b) ARMSE of phase error estimates versus SNR; (c) ARMSE of position error estimates versus SNR. and position error estimations cannot be separated in the conventional method and they interfere with each other. However, in the modified method, because gain errors are corrected before estimating position errors, position error estimates can converge to optimal solutions. The position error estimate ARMSE is lower than $0.01 \mathrm{~m}$ when the SNR is larger than $15 \mathrm{~dB}$. In addition, it is shown in Figure $2 \mathrm{~b}$ that phase error estimations can work well for both methods. This is consistent with the analysis in modified method for DSS-SAR system, which shows that position errors have no influence on the phase error estimation when the zero Doppler bin is chosen. However, the computational load of the modified algorithm is lower than the conventional method.

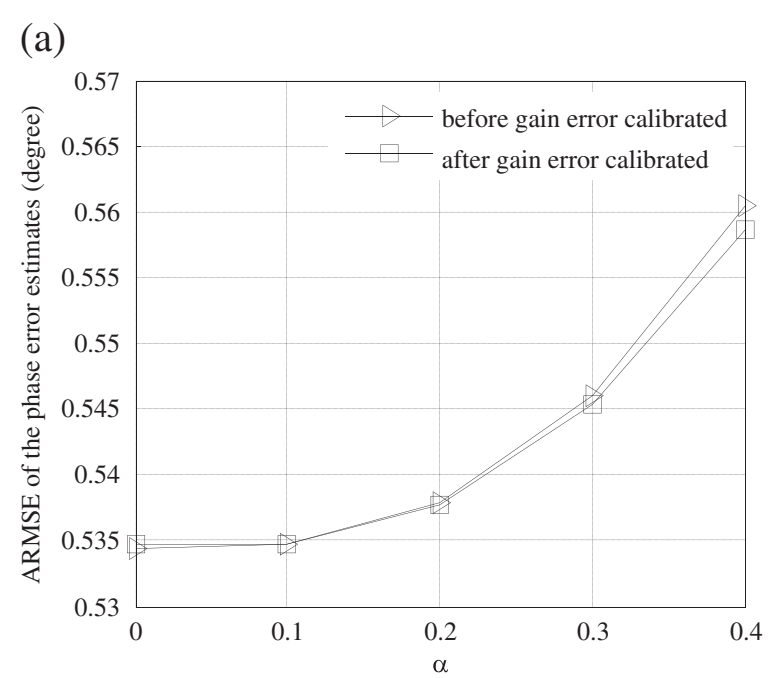

(b)

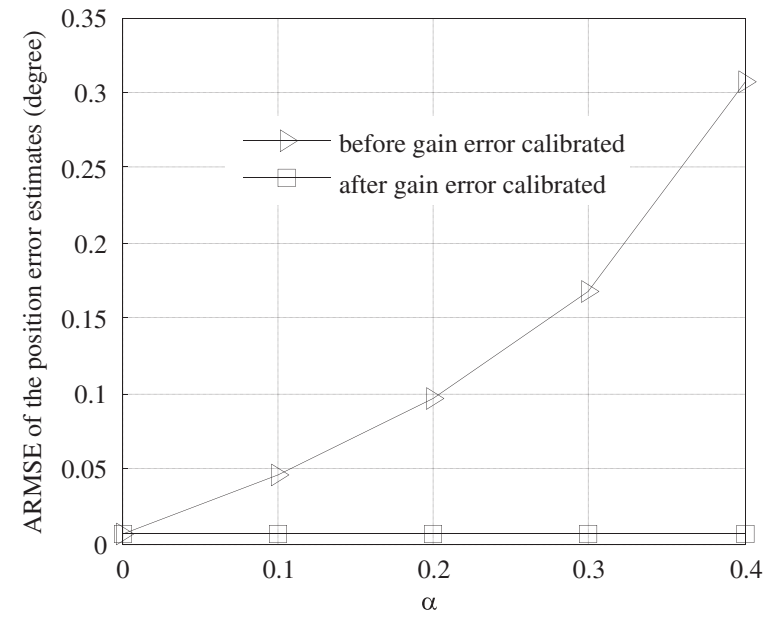

Figure 3 ARMSE of phase and position error estimates versus gain error by the modified method. (a) ARMSE of phase error estimates versus gain error; (b) ARMSE of position error estimates versus gain error. 


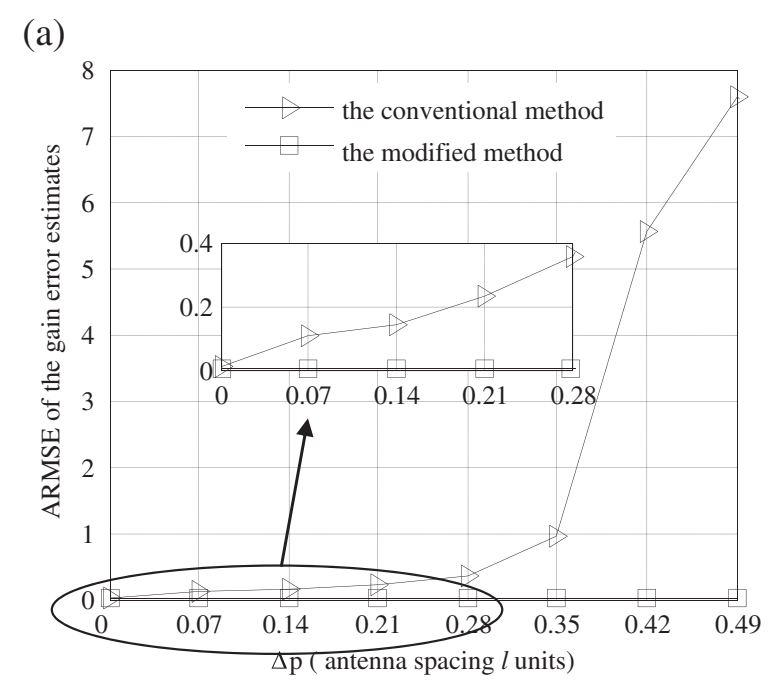

(b)

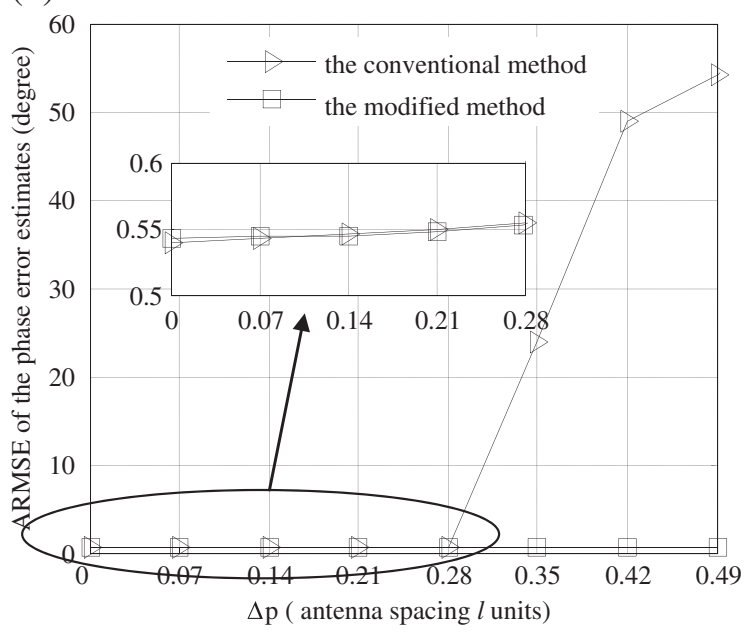

(c)

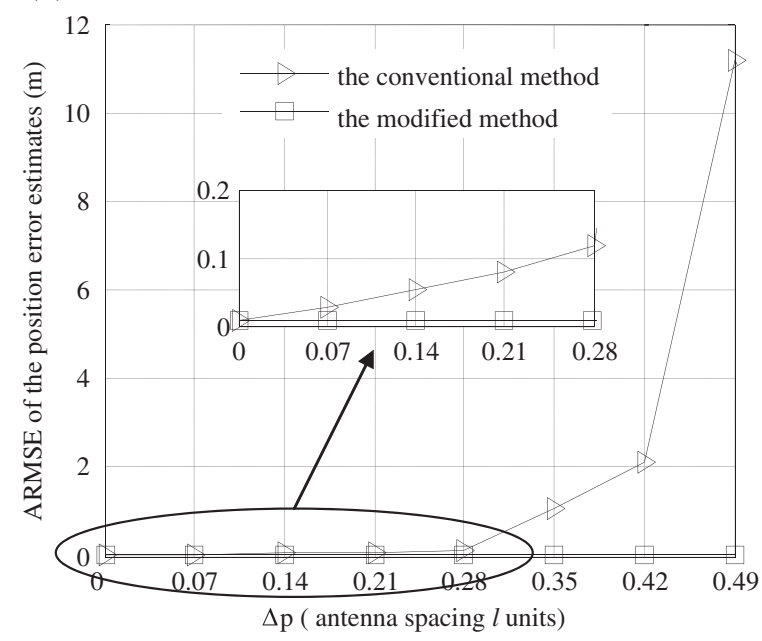

Figure 4 ARMSE of gain, phase and position error estimates versue position error. (a) ARMSE of gain error estimates versus position error; (b) ARMSE of phase error estimates versus position error; (c) ARMSE of position error estimates versus position error. (a)

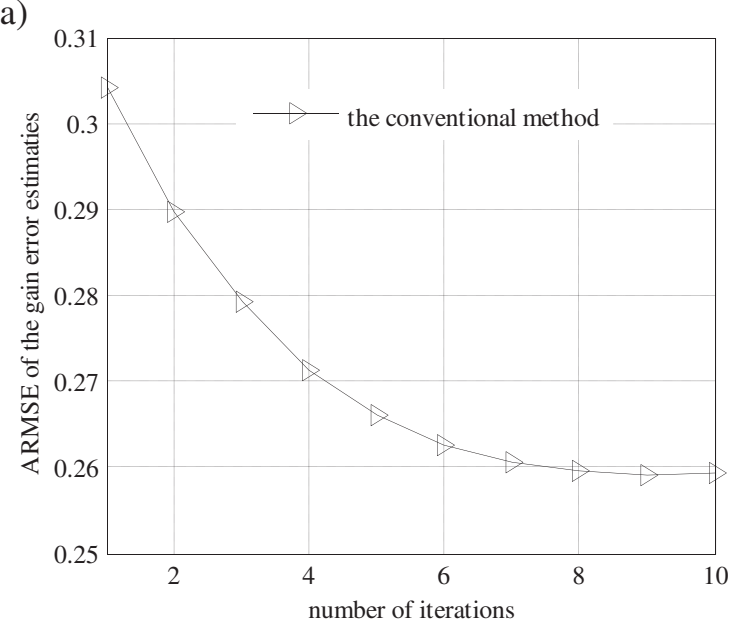

(b)

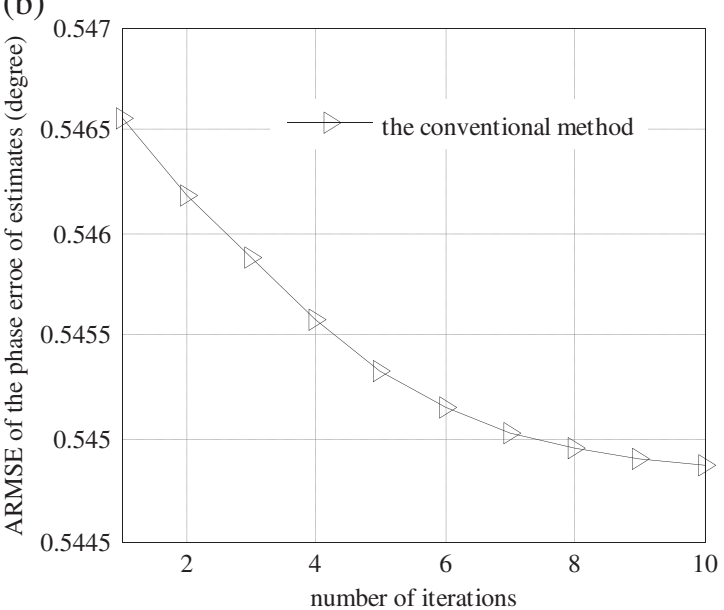

(c)

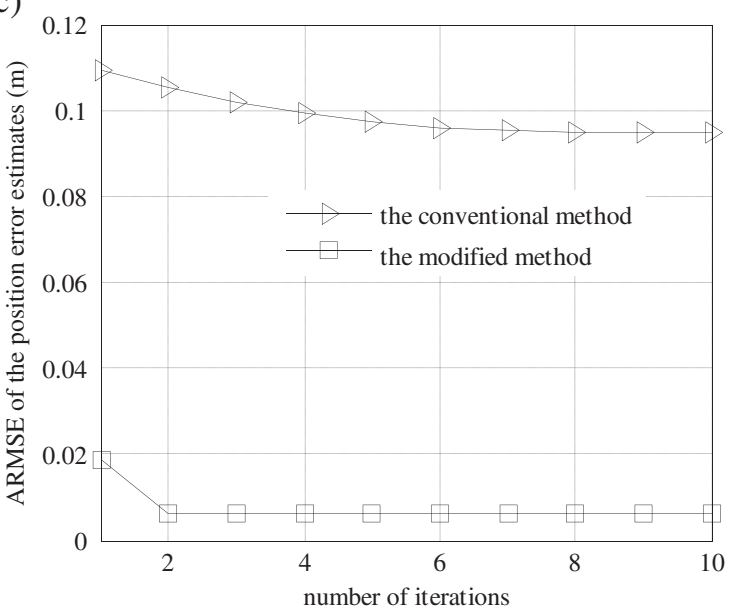

Figure 5 ARMSE of gain, phase and position error estimates versus number of iterations. (a) ARMSE of gain error estimates versus number of iterations; (b) ARMSE of phase error estimates versus number of iterations; (c) ARMSE of position error estimates versus number of iterations. 


\subsection{Effect of gain errors}

In the simulation, $\alpha$ changes from 0 to $0.4 . \Delta p=0.25 l$. SNR is $20 \mathrm{~dB}$. The other parameters in the simulation are the same as the former. The effect of gain estimation errors on the following phase and position error estimations in the modified method is given in Figure 3, where the curve gained without calibrating gain errors is denoted as "before gain error calibrated" while the other curve named "after gain error calibrated" is obtained by calibrating gain errors first. It can be seen that if gain errors cannot be well calibrated, the position error estimation difference becomes larger with the increment of $\alpha$, while the change of phase error estimation can be ignored. By using the modified method, both of phase and position error estimations can work well. It is clear that gain errors have serious influence on the following position error estimation and it is necessary to estimate gain errors first.

\subsection{Effect of position errors}

Fixing SNR to $20 \mathrm{~dB} . \alpha=0.2$. ARMSE curves of gain, phase, and position error estimates versus position error are shown in Figure $4 \mathrm{a}-\mathrm{c}$, respectively. Local simulation results (small figures embedded in Figure 4) are also given for better explanation. Being consistent with the analysis in modified method for DSS-SAR system, gain and position error estimations interfere with each other, which make gain and position error estimations converge to suboptimal solutions in the conventional method. So, the estimation accuracy of the conventional method cannot satisfy the requirement. The gain error estimate ARMSE is larger than 0.1 while the position error estimate ARMSE is close to 0.03 when $\Delta p=0.07 l$. In addition, phase error estimates obtained by both of the conventional method and the modified method are approximately equal to each other when $\Delta p \leq 0.28 l$. This is consistent with the previous analysis. By using the data of the zero Doppler bin, phase errors can be estimated with existing position errors. However, when $\Delta p \geq 0.35 \mathrm{l}$, the conventional method fails to work since gain and position error estimates deviate badly from the true values which has a strong impact on the phase error estimation.

\subsection{Effect of iterations}

Fix $\alpha=0.2, \Delta p=0.25 l$ and suppose SNR to be $20 \mathrm{~dB}$. The gain, phase, and position error estimation performance of the conventional method versus the number of iterations is shown in Figure $5 \mathrm{a}-\mathrm{c}$, respectively. It is observed from Figure $5 \mathrm{a}, \mathrm{c}$ that the conventional method converges slowly and even to suboptimal solutions since gain and position error estimations can interact with each other. However, it is noticed from Figure $5 \mathrm{~b}$ that the phase error estimation can work well as we analyzed before.
For the modified method, only the position error estimate ARMSE versus the number of iterations is shown in Figure $5 \mathrm{c}$ since only the position error estimation is iterated in the modified method. It is noticed from Figure $5 \mathrm{c}$ that the position error estimation converges to the optimal solution with less than three iterations. To sum up, the computational load of the modified method is less than that of the conventional method. Meanwhile better performance can be obtained by the modified method.

\section{Conclusions}

In this study, we focus on gain, phase, and position error estimations of DSS-SAR systems. Based on the conventional method, a modified array error estimation method is proposed here. In the conventional method, the estimation of gain and position errors may converge to suboptimal solutions especially when position errors are large and the joint iteration between the gain-phase and position error estimations are needed. That is because of the interaction between the estimations of gain and position errors. In the modified method, gain errors are first estimated and compensated before the other errors' estimation, which guarantees the position error estimation accuracy to be higher than that in the conventional method. Meanwhile, by using the zero Doppler bin data, the phase error estimation can behave independently of position errors. Then the joint iteration strategy between the gain-phase and position error estimations in the conventional method can be avoided, which makes the modified method perform stably with low computational load. Theoretical analysis and simulation results demonstrate the effectiveness of the modified method.

\section{Competing interests}

The authors declare that they have no competing interests.

\section{Acknowledgment}

The authors would like to thank the anonymous reviewers for their constructive comments, which led to significant improvements in this study. Special thanks to Dr. Yinghua Wang and Xuefeng Zheng for improving the English usage and also for their valuable suggestions. This study was sponsored in part by the National Basic Research Program of China (973 Program) under Grant no. 2011CB707001, in part by the Natural Science Foundation of China under Grant no. 61101242, in part by the Fundamental Research Funds for the Central Universities under Grant no. K50511020009, and in part by the Program for Changjiang Scholars and Innovative Research Team in University under Grant no. IRT0954.

Received: 19 June 2012 Accepted: 7 December 2012

Published: 22 February 2013

\section{References}

1. D Zhen, C Bin, L Diannong, Detection of ground moving targets for twochannel spaceborne SAR-ATI. EURASIP J. Adv. Signal Process., Article ID 230785 (2010). doi:10.1155/2010/230785

2. F Lombardini, M Pardini, G Fornaro et al, Linear and adaptive spaceborne three-dimensional SAR tomography: a comparison on real data. IET Radar Sonar Navigat. 3(4), 424-436 (2009)

3. P Wolfgang, M David, The Terra SAR-X satellite. IEEE Trans. Geosci. Remote Sens. 48(2), 615-622 (2010) 
4. S Chiu, MV Dragosevic, Moving target indication via RADARSAT-2 multichannel synthetic aperture radar processing. EURASIP. J. Adv. Signal Process., (2010). doi:10.1155/2010/740130

5. K Tomiyasu, Image processing of synthetic aperture radar range ambiguous signals. IEEE Trans. Geosci. Remote Sens. 32(5), 1114-1117 (1994)

6. NA Goodman, SC Lin, D Rajakrishna, JM Stiles, Processing of multiple receiver, spaceborne arrays for wide-area SAR. IEEE Trans. Geosci. Remote Sens. 40(4), 841-852 (2002)

7. S Li, HP Xu, LQ Zhang, An advanced DSS-SAR InSAR terrain height estimation approach based on baseline decoupling. Prog. Electromagn. Res. 119, 207-224 (2011)

8. CH Gierull, D Cerutti-Maori, J. Ender, Ground moving target indication with tandem satellite constellations. IEEE Geosci. Remote. Sens. Lett. 5(4), 710-714 (2009)

9. JP Aguttes, The SAR train concept: required antenna area distributed over $\mathrm{N}$ smaller satellites, increase of performance by $\mathrm{N}$, in Proceedings of the IGARSS'03, Toulouse, France (, 2003), pp. 542-544

10. ZF Li, HY Wang, T Su, Z Bao, Generation of wide-swath and high-resolution SAR images from multichannel small spaceborne SAR Systems. IEEE Geosci. Remote. Sens. Lett. 2(1), 82-86 (2005)

11. Z Lei, Q Cheng-Wei, X Mengdao, B Zheng, SAR imaging and Doppler ambiguity removal with distributed microsatellite arrays. Int. J. Remote. Sens. 31(24), 6441-6458 (2010)

12. ZF Li, Z Bao, HY Wang, GS Liao, Performance improvement for constellation SAR using signal processing techniques. IEEE Trans. Aerosp. Electron. Syst. 42(2), 436-451 (2006)

13. AJ Weiss, B Friedlander, DOA and steering vector estimation using a partially calibrated array. IEEE Trans. Aerosp. Electron. Syst. 32(3), 1047-1057 (1996)

14. KV Stavropoulos, A Manikas, Array calibration in the presence of unknown sensor characteristics and mutual coupling, in Proceedings of the EUSIPCO, Toulouse, France (, 2000), pp. 1417-1420

15. L Aifei, L Guisheng, Z Cao et al, An eigenstructure method for estimating DOA and sensor gain-phase errors. IEEE Trans. Signal Process. 59(12), 5944-5956 (2011)

16. B Friedlander, AJ Weiss, Direction finding in the presence of mutual coupling. IEEE Trans. Antennas Propagat. 39(3), 273-284 (1991)

17. AJ Weiss, B Friedlander, Array shape calibration using sources in unknown locations-a maximum likelihood approach. IEEE Trans. Acoust. Speech Signal Process. 37(12), 1958-1966 (1989)

18. MP Wylie, S Roy, RF Schmitt, Self-calibration of linear equi-spaced (LES) arrays, in Proceedings of the ICASSP-93, Minneapolis, USA (, 1993), pp. 281-284

doi:10.1186/1687-6180-2013-31

Cite this article as: Xu et al: Self-calibration method without joint iteration for distributed small satellite SAR systems. EURASIP Journal on Advances in Signal Processing 2013 2013:31.

\section{Submit your manuscript to a SpringerOpen ${ }^{\circ}$ journal and benefit from:}

- Convenient online submission

- Rigorous peer review

- Immediate publication on acceptance

- Open access: articles freely available online

- High visibility within the field

- Retaining the copyright to your article

Submit your next manuscript at $\gg$ springeropen.com 